\title{
Interaction Effects of 3-Mercaptohexan-1-ol (3MH), Linalool and Ethyl Hexanoate on the Aromatic Profile of South African Dry Chenin Blanc Wine by Descriptive Analysis (DA)
}

\author{
C. Wilson ${ }^{1 \#}$, J. Brand ${ }^{1}$, W. du Toit ${ }^{1}$, A. Buica ${ }^{1,2 *}$ \\ (1) Department of Viticulture and Oenology, Stellenbosch University, South Africa \\ (2) Institute for Grape and Wine Sciences, Stellenbosch University, South Africa
}

Submitted for publication: January 2018

Accepted for publication: April 2018

Key words: Interaction effects, Chenin Blanc, descriptive analysis, sensory evaluation, wine matrix

\begin{abstract}
Interaction studies are some of the most interesting sensory experiments that highlight the effect of composition on wine perception. The use of single compounds, viz. an ester (ethyl hexanoate), a terpene (linalool) and a thiol (3-mercaptohexanol, 3MH), which have previously been shown to be representative of Chenin Blanc wines, resulted in typical descriptors for these compounds, such as 'apple, 'floral' and 'guava' respectively. Interaction effects were observed between the compounds, and these were reflected in both the nature and the level of attributes generated. Additionally, interaction effects between the compounds (singles and combinations) and the wine matrix indicated that the latter plays an important role in the perception of wine aromas. The use of a dearomatised neutral wine base added an extra dimension to this study, which usually is done in a simpler matrix, such as a model wine.
\end{abstract}

\section{INTRODUCTION}

Recent research has established that both 3-mercaptohexan1-ol (3MH) and 3-mercaptohexyl acetate (3MHA) can be present in South African Chenin Blanc wines at concentrations many times higher than their odour thresholds (Wilson, 2016). 3-Mercaptohexan-1-ol has an odour threshold of $60 \mathrm{ng} / \mathrm{L}$ and is described as 'passion fruit' and 'grapefruit' (Tominaga et al., 1998). These descriptors are supported by the fact that $3 \mathrm{MH}$ has been identified in passion fruit itself (Engel \& Tressl, 1991). 3-Mercaptohexyl acetate has an odour threshold of $4.2 \mathrm{ng} / \mathrm{L}$ and is best described as 'box tree' (also known as 'box hedge'), but also as 'grapefruit' and 'passion fruit' (Tominaga et al., 1996; Dubourdieu et al., 2006), as well as 'guava' and 'gooseberry' (Swiegers \& Pretorius, 2007). Somewhat problematically, 'box tree' is a culturally specific term unfamiliar in the South African context, where 'guava' and 'gooseberry' are more likely to be used. While typical descriptors for $3 \mathrm{MH}$ and $3 \mathrm{MHA}$ are known, that knowledge alone cannot be used to predict the aromatic expression of these compounds in the context of wine. This is because, firstly, the sensory perception of volatiles changes in intensity (López et al., 2003) and character (Fretz et al., 2005; Mateo-Vivaracho et al., 2010; Van Wyngaard et al., 2014; Coetzee et al., 2015) at different concentrations. Secondly, volatile aromatic compounds do not exist in isolation, but rather form a small component of the complex wine matrix. This matrix includes over
1000 other volatiles, which can interact with one another and thereby affect the sensory perception (Polášková et al., 2008). It is for this reason that one of the first studies on the 'guava' character of Chenin Blanc by Van Rooyen et al. (1982) suggested "...observing the effect on the guava-like character in neutral wines by altering their composition ... By changing one or two factors at a time, further evidence could be collected for a better understanding of the phenomenon". Similar calls for interaction studies have been reiterated by other wine aroma researchers (Francis \& Newton, 2005; Polášková et al., 2008).

Some researchers have addressed this by performing interaction studies. A few such studies involved thiols, although these studies were designed to be relevant to Sauvignon Blanc wines (King et al., 2011; Benkwitz et al., 2012; Van Wyngaard et al., 2014; Coetzee et al., 2015). These studies show the enhancing and suppressing effects that volatiles can have on one another. For example, in one experiment it was found that 3MHA reduces the 'sweet', 'floral' and 'muscat' character of linalool and 2-phenylethyl acetate, while methoxypyrazines reduce the tropical intensity of 3MHA (Campo et al., 2005). Similar antagonistic interactions between $3 \mathrm{MH}$ and pyrazines have been seen in other interaction studies (Van Wyngaard et al., 2014; Coetzee et al., 2015). To our knowledge, no interaction studies with a focus on Chenin Blanc have been published.

\footnotetext{
\# Current affiliation: E\&J Gallo, Modesto, California, USA

*Corresponding author: E-mail address: abuica@sun.ac.za

Acknowledgments: The authors would like to thank Winetech and THRIP, for financial support
} 
Three compounds present in Chenin Blanc wines are $3 \mathrm{MH}$, ethyl hexanoate, and linalool (Lawrence, 2012). 3MH is typically described as 'passion fruit' and 'grapefruit' (Tominaga et al., 1998), although in recent interaction studies these descriptors have been expanded to include 'guava' and 'green' aromas (King et al., 2011; Van Wyngaard et al., 2014; Coetzee et al., 2015). Ethyl hexanoate has aromas of 'apple peel' and 'fruit' in wine (Francis \& Newton, 2005) and an odour threshold of $14 \mu \mathrm{g} / \mathrm{L}$ (Ferreira et al., 2000). It was previously suggested as a possible source for the 'guava' aroma of Chenin Blanc wines (Van Rooyen et al., 1982). Both ethyl hexanoate and $3 \mathrm{MH}$ are odorants that have been found in guava fruit (Steinhaus et al., 2009; Pino \& Bent, 2013). Linalool is best known for giving a 'floral' character to Muscat wine varieties (Mateo \& Jiménez, 2000), but also has aromas of 'citrus' and 'lavender' (Black et al., 2015) and an odour threshold of $25.2 \mu \mathrm{g} / \mathrm{L}$ (Ferreira et al., 2000). Although ethyl hexanoate and linalool oxide have been shown to differentiate between different styles of South African Chenin Blanc wines (Lawrence, 2012), the role of thiols in these wines has only recently been studied (Wilson, 2016). Furthermore, the interactions between these three compounds in Chenin Blanc are not known.

In this work, an interaction experiment was performed by spiking a partially dearomatised Chenin Blanc wine with combinations of $3 \mathrm{MH}$, ethyl hexanoate and linalool at various concentrations. Samples were spiked with each individual compound, as well as with combinations of all three. The sensory method used to analyse these samples was descriptive analysis (DA). DA is well suited to quantifying small differences between products by training a panel to be able to rate differences in the intensity of descriptors (Lawless \& Heymann, 2010). The intensity rating allows for the observation of the enhancing and suppressing effects of the three compounds on one another. The comparison of these compounds alone and in combination will allow for the description of these three compounds in the South African Chenin Blanc matrix, and the identification of any enhancing or suppressing effects they may have on one another. Studying the sensory perception of these compounds would help further understand the role of thiols in the context of South African Chenin Blanc wines.

\section{MATERIALS AND METHODS \\ Experimental design}

Two different descriptive analysis (DA) experiments were performed by the same judges. The first experiment was an interaction study evaluating the three compounds in combination at three different levels, and the second experiment evaluated the same compounds separately. These will be referred to as "combinations" and "singles" respectively. Only the aroma of the samples was evaluated.

Low, medium and high levels of ethyl hexanoate $(600 \mu \mathrm{g} / \mathrm{L}, 1100 \mu \mathrm{g} / \mathrm{L}$ and $1600 \mu \mathrm{g} / \mathrm{L})$ and linalool $(200 \mu \mathrm{g} / \mathrm{L}$, $1600 \mu \mathrm{g} / \mathrm{L}$ and $3000 \mu \mathrm{g} / \mathrm{L}$ ) were selected according to published Chenin Blanc chemical analysis data (Lawrence, 2012). The $3 \mathrm{MH}$ levels selected (200 ng/L, $1100 \mathrm{ng} / \mathrm{L}$ and $2000 \mathrm{ng} / \mathrm{L}$ ) are within the range typically found in South African Chenin Blanc wines (Wilson, 2016). At all levels, the compounds were present at concentrations above their odour thresholds, and their maximum odour active values were 114 for ethyl hexanoate, 119 for linalool, and 33 for $3 \mathrm{MH}$. The same levels were used for both the combinations and singles.

Since a full factorial design would have resulted in 27 samples for the sensory analysis of the combinations, a central composite design (CCD) was used to reduce the sample set to 16 (Table 1), as proposed by Esbensen (2002). The Unscrambler ${ }^{\circledR}$ X (Version 10.2) was used to generate a small inward-facing central composite design with six axial samples, eight cube samples and two centre samples. For the singles, each level of each compound was spiked on its own, giving nine samples in total (Table 2).

\section{Samples}

A dry, unwooded, commercially available Chenin Blanc wine was selected based on its neutral aroma, and it was treated to obtain a partially dearomatised base wine. During the treatment and blending steps, the wine was protected from oxidation under $\mathrm{N}_{2}$ gas. The wine was dearomatised with $5 \mathrm{~g} / \mathrm{L}$ activated charcoal powder (Merck, Darmstadt, Germany) for seven hours without agitation, then separated from the charcoal by diatomaceous earth filtration. In a screening session, three researchers chose a blend of $1 / 3$ charcoal-treated wine to $2 / 3$ untreated wine that yielded a neutral wine base with low aromatic intensity.

Dilutions of pure 3MH (98\%, Interchim, Montluçon, France), ethyl hexanoate ( $\geq 99 \%$, Sigma-Aldrich, St. Louis, Missouri), and $( \pm$ ) linalool (97\%, Sigma-Aldrich) for spiking were prepared in HPLC-grade ethanol $(\geq 99.8 \%$, SigmaAldrich) and stored at $-80{ }^{\circ} \mathrm{C}$ for no more than 5 weeks. All samples were prepared by spiking the partially dearomatised base wine for 12 hours prior to training or testing (no more than $1 \mathrm{~mL}$ spiking solution to $750 \mathrm{~mL}$ base wine), during which time the samples were stored under $\mathrm{N}_{2}$ gas at $4{ }^{\circ} \mathrm{C}$. The delay between spiking time and evaluation allowed for the integration of the aroma compounds into the matrix. Samples were allowed one hour to reach room temperature before being served. The levels of $3 \mathrm{MH}$ spiked were checked according to the method of Piano et al. (2015), and the ethyl hexanoate and linalool levels were checked in terms of the methods detailed in Wilson (2016).

\section{Sensory evaluation \\ Panellists}

The same panel of ten judges aged 23 to 58 years (one male, nine females) participated in both experiments. The judges were members of the community, as well as students and staff of the Department of Viticulture and Oenology at Stellenbosch University. The panellists were recruited based on their willingness to participate and previous experience evaluating South African Chenin Blanc, and were remunerated for their participation. Eight of the ten panellists had previous experience with the analysis of Chenin Blanc wines by descriptive analysis.

\section{Combinations}

The judges were not informed of the nature or goal of the study. The aroma of the spiked, partially dearomatised wine was evaluated over 10 one-hour training sessions spanning 
TABLE 1

Central composite design of combinations showing sample codes and spiking levels. Level 1=low, level 2=medium, level $3=$ high.

\begin{tabular}{|c|c|c|c|c|c|c|c|}
\hline CCD Name & Sample Name & $\begin{array}{c}\text { Factor } 1 \\
\text { level }\end{array}$ & $\begin{array}{c}\text { Factor } 2 \\
\text { level }\end{array}$ & $\begin{array}{c}\text { Factor } 3 \\
\text { level }\end{array}$ & $\begin{array}{l}3 \mathrm{MH} \\
(\mathrm{ng} / \mathrm{L})\end{array}$ & $\begin{array}{l}\text { ethyl hexanoate } \\
(\mu \mathrm{g} / \mathrm{L})\end{array}$ & $\begin{array}{l}\text { linalool } \\
(\mu \mathrm{g} / \mathrm{L})\end{array}$ \\
\hline Axial_A (high) & $3 \_2 \_2$ & 3 & 2 & 2 & 2000 & 1100 & 1600 \\
\hline Axial_A (low) & $1 \_2 \_2$ & 1 & 2 & 2 & 200 & 1100 & 1600 \\
\hline Axial_B (high) & $2 \_3 \_2$ & 2 & 3 & 2 & 1100 & 1600 & 1600 \\
\hline Axial_B (low) & 2_1_2 & 2 & 1 & 2 & 1100 & 600 & 1600 \\
\hline Axial_C (high) & $2 \_23$ & 2 & 2 & 3 & 1100 & 1100 & 3000 \\
\hline Axial_C (low) & $2 \_2 \_1$ & 2 & 2 & 1 & 1100 & 1100 & 200 \\
\hline Cube1 & 1_1_1 & 1 & 1 & 1 & 200 & 600 & 200 \\
\hline Cube2 & 3_1_1 & 3 & 1 & 1 & 2000 & 600 & 200 \\
\hline Cube3 & $1 \_3 \_1$ & 1 & 3 & 1 & 200 & 1600 & 200 \\
\hline Cube4 & 3_3_1 & 3 & 3 & 1 & 2000 & 1600 & 200 \\
\hline Cube5 & $1 \_1 \_3$ & 1 & 1 & 3 & 200 & 600 & 3000 \\
\hline Cube6 & $3 \_1 \_3$ & 3 & 1 & 3 & 2000 & 600 & 3000 \\
\hline Cube 7 & $1 \_3 \_3$ & 1 & 3 & 3 & 200 & 1600 & 3000 \\
\hline Cube8 & $3 \_3 \_3$ & 3 & 3 & 3 & 2000 & 1600 & 3000 \\
\hline сp01 & 2_2_2 & 2 & 2 & 2 & 1100 & 1100 & 1600 \\
\hline cp02 & $2 \_2 \_$ & 2 & 2 & 2 & 1100 & 1100 & 1600 \\
\hline
\end{tabular}

TABLE 2

Sample codes and spiking levels of single compounds. $\mathrm{H}=3 \mathrm{MH}, \mathrm{E}=$ ethyl hexanoate, $\mathrm{L}=$ linalool.

\begin{tabular}{lccc}
\hline Sample Name & $3 \mathrm{MH}(\mathrm{ng} / \mathrm{L})$ & Ethyl hexanoate $(\mu \mathrm{g} / \mathrm{L})$ & linalool $(\mu \mathrm{g} / \mathrm{L})$ \\
\hline H_low & 200 & 0 & 0 \\
H_med & 1100 & 0 & 0 \\
H_high & 2000 & 0 & 0 \\
E_low & 0 & 600 & 0 \\
E_med & 0 & 1100 & 0 \\
E_high & 0 & 0 & 2000 \\
L_low & 0 & 0 & 1600 \\
L_med & 0 & 0 & 3000 \\
L_high & 0 & 0 &
\end{tabular}

a period of three weeks. Each training session alternated between the axial and cube samples to minimise sensory fatigue. During consensus training, descriptors generated by the panellists were defined using aroma reference standards. The use of references helped to familiarise all the panellists with the terms and to standardise their understanding of the descriptors. Initially, 34 reference standards were presented. Throughout the training process, the lexicon was narrowed to 18 descriptors by the panel (Table 3 ).
For the testing sessions, spiked wines were poured in 20 $\pm 2 \mathrm{~mL}$ aliquots into clear glasses (ISO NORM, 3591:1977) one hour before serving and covered with plastic Petri dish lids. Each glass was labelled with a unique, random threedigit code. All evaluations took place in off-white individual sensory booths in a quiet, well-ventilated, odourless $20 \pm 2{ }^{\circ} \mathrm{C}$ air-conditioned room (ISO NORM, 8589:2007). The sixteen samples were presented in a monadic sequential manner according to a Williams Latin Square design (MacFie et al., 
1989). The sample set was divided into subsets of five or six glasses, and the panellists were given a 15-minute break between subsets to minimise sensory fatigue. Panellists rated the intensity of each descriptor along an unstructured line scale from "none" to "intense" using Compusense ${ }^{\circledR}$ five software (Release 5.6). Two panellists preferred to use paper rather than a computer to rate the samples and were allowed to do so. Four replications of the combinations were performed, each on a separate day.

\section{Singles}

After evaluation of the combinations, the same panel received one training session to practise evaluating samples that were spiked with only one level of one compound at a time. Only one training session was deemed necessary because the singles were inherently less complex, and the descriptors generated and reference standards used for these samples were the same as for the combinations (Table 3). Thus, the training for the combinations was deemed sufficient to evaluate the singles as well. Testing was performed following the same methods and procedures as for the combinations. Four replications of the singles set were performed over two days, with a fifteen-minute break between replications to avoid sensory fatigue.

\section{Statistical analyses}

The performance of the panel was evaluated using PanelCheck (V1.4.2), according to the workflow suggested by Tomic et al. (2010). The discriminability and consensus of the panel were evaluated by means of analysis of variance (ANOVA) and Tucker-1 plots. The data structure of both experiments - combinations and singles - was analysed by mixed-model ANOVA. For both experiments, the significance threshold was set at $p=0.05$. The Fisher's LSD post-hoc test was used to show significant differences. Response-surface plots were created to illustrate two-way interactions in Statistica (Version 13) by doing regression analyses in the way central composite design (CCD) data is analysed. Principal component analysis (PCA) was also performed to illustrate correlations between attributes and samples. PCA was run on the covariance matrices of both experiments in XLSTAT (Version 18.06, Addinsoft), as suggested by Borgognone et al. (2001). Descriptors included in the PCAs were limited to those with a significant main effect or significant interaction effect in the ANOVAs.

\section{RESULTS AND DISCUSSION}

The panel performance of both experiments was acceptable, as evaluated by the workflow described above (data not shown). Although the training and testing of the singles took place chronologically after the combinations, the results are presented in the opposite order to explain the attributes associated with the compounds before investigating the interaction between the compounds.

\section{Singles}

The panel was able to agree upon differences in aroma between the singles, shown by the very high $93.8 \%$ explained

TABLE 3

Reference standards and corresponding descriptors agreed upon by the panel for both experiments.

\begin{tabular}{|c|c|}
\hline Descriptor & Reference standard \\
\hline Guava & $3 \mathrm{~cm}^{3}$ fresh, ripe guava \\
\hline Pineapple & $2 \mathrm{~cm}^{3}$ fresh pineapple \\
\hline Passion fruit & $1 / 4$ of the pulp from a fresh passion fruit \\
\hline Banana & $1 \mathrm{~cm}^{3}$ ripe banana in $10 \mathrm{~mL}$ distilled water \\
\hline Peach & $3 \mathrm{~cm}^{3}$ canned peach ("Koo”) \\
\hline Apple & $3 \mathrm{~cm}^{3}$ fresh green apple with skin \\
\hline Lemon & $3 \mathrm{~cm}^{3}$ fresh fruit (pulp + flesh) \\
\hline Orange & $3 \mathrm{~cm}^{3}$ fresh fruit (pulp + flesh) \\
\hline Grapefruit & $3 \mathrm{~cm}^{3}$ fresh fruit (pulp + flesh) \\
\hline Floral & verbally agreed upon as an all-encompassing floral category \\
\hline Orange blossom & 2 drops solution "Ferminich" on a cotton ball \\
\hline Bergamot/Earl Grey & 1.5 g Earl Grey tea ("Five Roses") \\
\hline Tea & 1.5 g black tea ("Five Roses") \\
\hline Artificial sweet & $1 \mathrm{~g}$ cotton candy \\
\hline Honey & $5 \mathrm{~mL}$ honey $+10 \mathrm{~mL}$ hot water \\
\hline Dusty/mineral & small chip of slate stone, wetted with water \\
\hline Tomato leaf & fresh cherry tomato leaves and stalk \\
\hline Cooked vegetables & $5 \mathrm{~mL}$ canned green bean brine ("Koo") $+5 \mathrm{~mL}$ canned asparagus brine ("Food Lover's Signature") \\
\hline
\end{tabular}


variance in the PCA (Fig. 1). 3MH and linalool had a greater impact on aroma than ethyl hexanoate, as these compounds oppose one another along PC1 of the PCA, which explains $81.8 \%$ of the variance in the data. The samples spiked with ethyl hexanoate clustered closer to the centre of the PCA and did not explain much of the variation between samples. The descriptors that were not significantly different in intensity between the samples were 'pineapple', 'passion fruit', 'banana', 'artificial sweet' and 'honey'. The non-significant 'passion fruit' descriptor is of note, as it is one of the typical descriptors of $3 \mathrm{MH}$. 'Passion fruit' was perceived in all the samples at a similar intensity, though Coetzee et al. (2015) found that 'passion fruit' became the dominant descriptor of $3 \mathrm{MH}$ in model wine at concentrations above $2000 \mathrm{ng} / \mathrm{L}$. The descriptors with significant differences in intensity between samples were 'guava', 'peach', 'apple', 'lemon', 'orange', 'grapefruit', 'floral', 'orange blossom', 'bergamot/ Earl Grey', 'tea', 'dusty/mineral', 'tomato leaf', and 'cooked vegetables'.

The medium and high levels of $3 \mathrm{MH}(\mathrm{H}$ medium, $\mathrm{H}$ high) correlated with 'lemon' in the PCA, as well as with the thiol-related descriptors 'grapefruit', 'guava', 'tomato leaf' and 'cooked veg' (Fig. 1). The association of 3MH with 'tomato leaf' and 'guava' descriptors is in agreement with recent interaction studies in model wine (Coetzee et al., 2015) and dearomatised Sauvignon Blanc wine (Van Wyngaard et al., 2014). The strong effect of high $3 \mathrm{MH}$ on 'tomato leaf' and 'guava' intensity is visible in the spider web plot (Fig. 2). As shown by the graph of the LS means, 'guava' intensity increased at greater concentrations of $3 \mathrm{MH}$, and was significantly higher than all other samples in the $\mathrm{H}$ high sample (Fig. 3). This pattern is the same for 'tomato leaf', although it was rated at lower average intensities compared to 'guava' (Fig. 2). Additionally, 'guava' intensities were higher for samples with ethyl hexanoate than with linalool (Fig. 3), which could indicate either an enhancing effect on the 'guava' attribute by ethyl hexanoate or a suppressing effect by linalool. 'Grapefruit' intensity was also significantly higher in wines spiked with $3 \mathrm{MH}$ than with linalool, and was intermediate in wines spiked with ethyl hexanoate (result not shown). 'Cooked veg' was rated at lower intensities overall, but behaved similarly to 'guava', with the highest intensity in the H_high sample (Fig. 3). However, it was also high in the L_low and E_low samples. The relationship of 'cooked veg' and a similar 'cooked beans' attribute with thiols has been previously established (King et al., 2011; Coetzee et al., 2015). In the PCA, the H_medium sample moved toward the subtle ethyl hexanoate-spiked samples, and the $\mathrm{H}$ low sample was grouped with them (Fig. 1).

The correlation of 'lemon' with the $3 \mathrm{MH}$-spiked wines

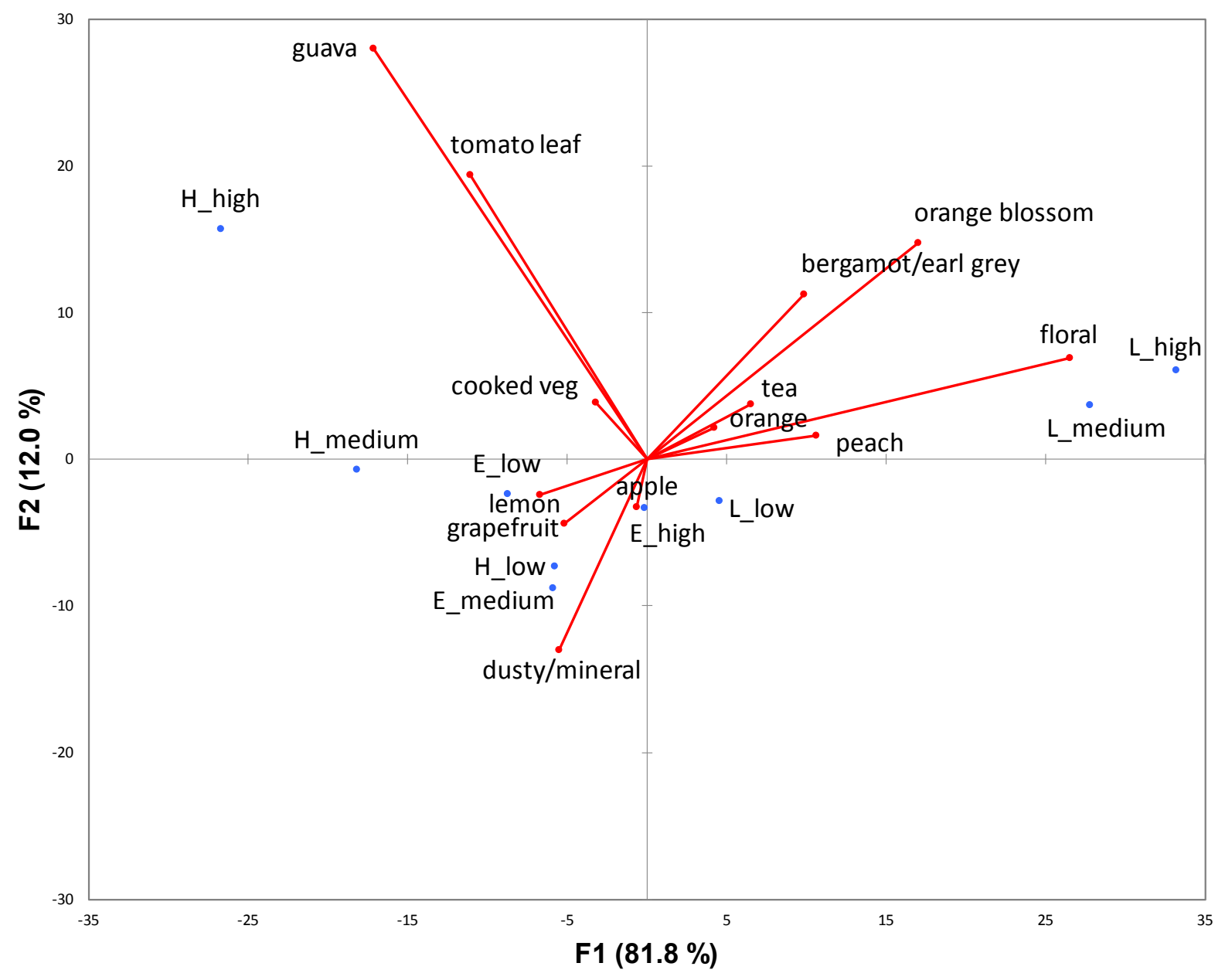

FIGURE 1

PCA of single compound data, showing attributes with a significant main or interaction effect. $\mathrm{H}=3 \mathrm{MH}, \mathrm{E}=\mathrm{ethyl} \mathrm{hexanoate}$, $\mathrm{L}=$ linalool. See Table 2 for spiking levels. 
in the PCA (Fig. 1) is misleading, as it was caused by a significant negative correlation of 'lemon' with the L_high, rather than a positive correlation with $3 \mathrm{MH}$ (Fig. 3). The same is true for 'dusty/mineral' (results not shown). Not explained well by $\mathrm{PC} 1$ or PC2 in the PCA, but relevant to the $3 \mathrm{MH}$-spiked samples, was the descriptor 'apple'. The case of the descriptor 'apple' was particularly complex, as it was affected by different concentrations of two compounds. For $3 \mathrm{MH}$, it reached the highest intensity in the $\mathrm{H}$ _medium sample (Fig. 3). Although ethyl hexanoate is described in the literature as 'apple peel' (Francis \& Newton, 2005), 'apple' was higher in the E_low than in the E_medium sample (Fig. 3).

All three levels of linalool-spiked samples (L high, L medium and L_low) in the PCA are positioned opposite $\mathrm{H}_{-}$ high, H_medium and $\mathrm{H} \_$low, and are highly correlated with the descriptors 'bergamot/Earl Grey', 'orange blossom', 'orange', 'tea' 'floral' and 'peach' (Fig. 1). In the ANOVA results for 'peach', 'tea' and 'orange', the compound main effect was significant, showing that, at all levels tested, linalool increased the intensity of 'peach', 'tea' and 'orange' aroma in the samples, but the intensity did not change significantly between different linalool levels (results not shown). A significant compound*level interaction for 'floral', 'orange blossom' and 'bergamot/Earl Grey' shows that the intensity of these descriptors increases with higher spiking levels. As monoterpenes are typically associated with 'floral' aromas, these results are in agreement with the existing literature (Marais, 1983). The descriptors correlated with linalool can be explained by the different aroma attributes of the two enantiomers in the racemic mixture of linalool. The (S) $(+)$-linalool enantiomer is 'citric' and is found in orange oils, while the (R)-(-)-linalool has a 'woody lavender' attribute and is found in lavender and bergamot oils (Padrayauttawat et al., 1997). 'Floral' intensity is lower in the H_medium and $\mathrm{H}$ _high samples, showing a potential suppressing effect of $3 \mathrm{MH}$, but this is only seen for the overall 'floral' descriptor (Fig. 3), and not the specific floral attributes. Linalool-spiked samples also had a significantly lower 'lemon' intensity than samples spiked with the other compounds, as well as a lower 'grapefruit' intensity than samples spiked with 3MH (Fig. 3), and a lower 'dusty/mineral' intensity in L_high than in L_ low (Fig. 2). In the case of 'cooked veg', it was highest in L_low, but L_medium and L_high had the lowest intensity (Fig. 3). However, it should be borne in mind that some of these differences are small compared to differences in the intensity ratings of certain other descriptors.

The sensory contribution of ethyl hexanoate is subtle when compared to the other two compounds, $3 \mathrm{MH}$ and linalool. While ethyl hexanoate is described in the literature as 'fruity' and 'green apple', it was not described by the 'apple' aroma in this study. In the case of 'floral'/'orange blossom' and 'guava'/'tomato leaf', in terms of which the $3 \mathrm{MH}$-spiked and linalool-spiked samples differed greatly, ethyl hexanoate-spiked samples had medium intensities of all attributes (Fig. 2). The restrained effect of ethyl hexanoate on aroma could be similar to the behaviour of another ester, 2-phenylethyl acetate, found by Campo et al. (2005), where it had to be in combination with compounds of similar aroma character to have an impact.

In summary, higher levels of $3 \mathrm{MH}$ increase the perception of 'guava', 'tomato leaf' and 'cooked veg', and may suppress 'floral'. Samples spiked with linalool are described with 'peach', 'tea', 'orange', and 'floral' descriptors,

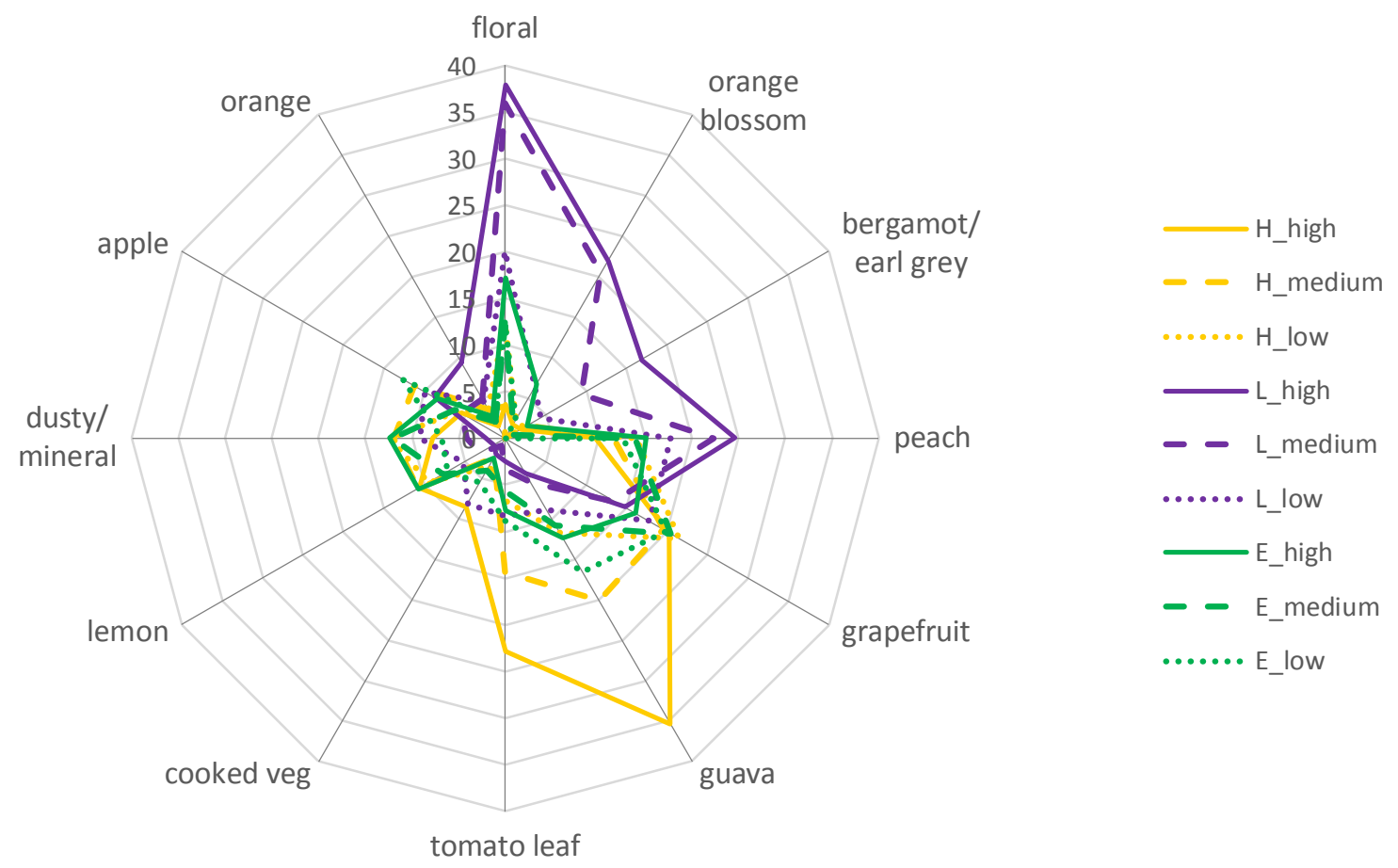

FIGURE 2

Spider web interaction showing the aromatic profile of the single DA samples for descriptors with a significant compound main effect or a significant compound*level interaction at $\alpha=0.05$. 

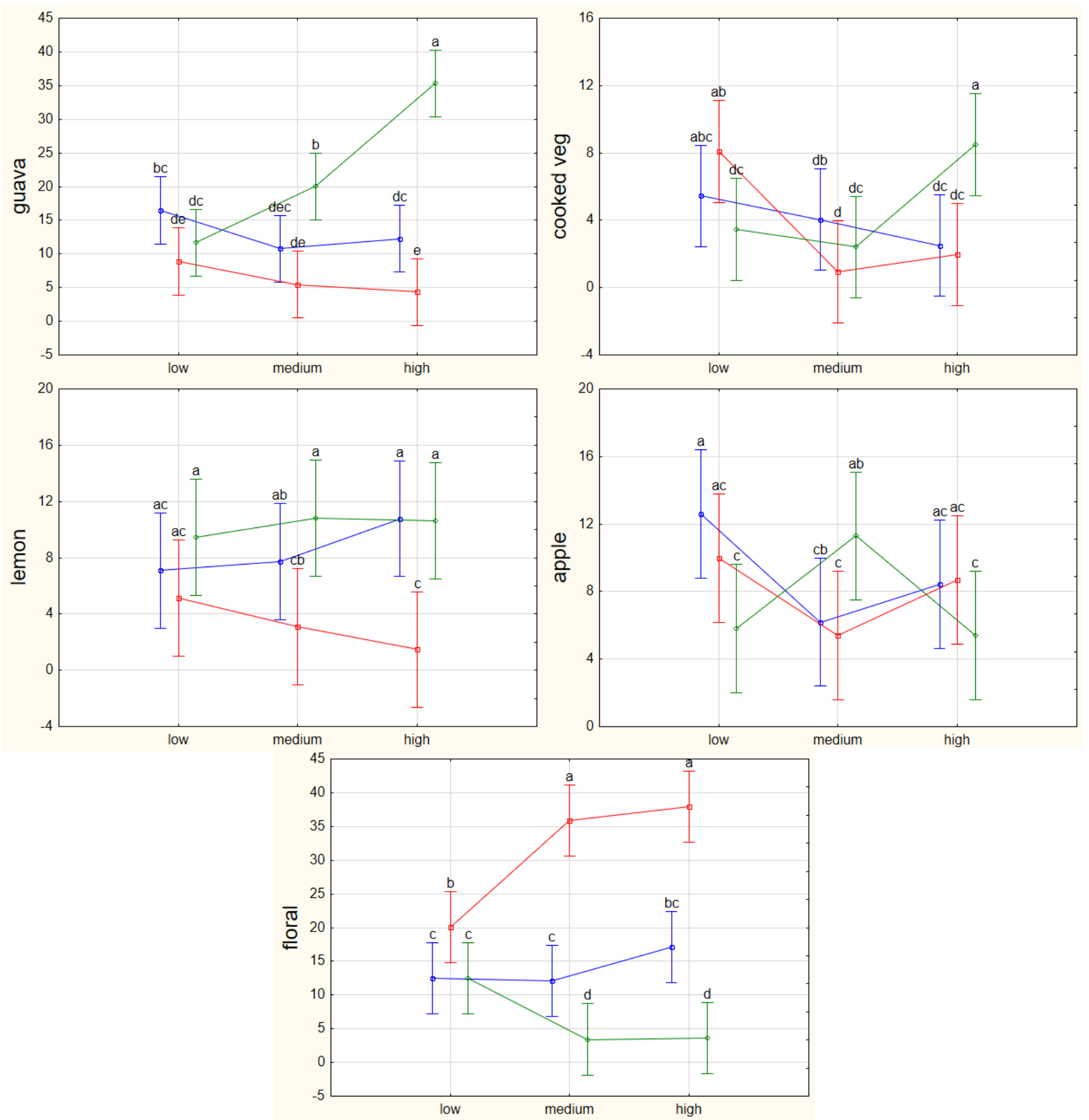

FIGURE 3

LS means plot illustrating the compound*level interaction effect on 'guava', 'cooked vegetables', 'apple', 'lemon' and 'floral' aroma intensity for the single compounds, with significant letters from the LSD post-hoc test. Vertical bars denote $95 \%$ confidence intervals. The compounds are colour coded: ethyl hexanoate (blue), linalool (green), 3MH (red).

including the specific floral attributes 'bergamot/Earl Grey' and 'orange blossom'. Linalool decreases the intensity of 'lemon' and 'grapefruit' and, at high concentrations, decreases 'dusty/mineral' and 'cooked veg'. It is interesting that, of the citrus descriptors, linalool increases 'orange', but decreases 'lemon' and 'grapefruit', so in this case rating only a general 'citrus' descriptor would have resulted in a loss of information. The highest mean intensities of all the descriptors are for 'guava', at high $3 \mathrm{MH}$ levels, and 'floral', at high linalool levels. The sensory contribution of ethyl hexanoate is minimal compared to the aromatic power of the thiol and the terpene. These results are compared to the combinations to see how the perception of these compounds changes when in solution with one another.

\section{Combinations}

The sample codes used in the PCA and spider plot can be found in Table 1, and follow the format $1=$ low, $2=$ medium and $3=$ high level of each compound in the order: $3 \mathrm{MH}_{-}$ ethyl hexanoate linalool. The panellists found it more difficult to evaluate the combinations than the singles. Not only were these samples more aromatically complex, but some panellists communicated that the aromas evolved quickly in the headspace of the glass, posing a challenge 
during evaluation. To address this, the panellists were instructed to use their initial impression of the aroma to rate the samples. This change in difficulty and complexity is shown by a decrease in explained variance, from $91 \%$ in the PCA of the singles (Fig. 1) to $67.9 \%$ in the PCA of the combinations (Fig. 4). It is further supported by the fact that the two centre samples, 2_2_2, are not very closely located on the PCA of the combinations (Fig. 4). Considering that the panel was able to differentiate between the samples in the singles, this can be attributed to the complexity of the samples, rather than to panel performance. There were also fewer significant descriptors than in the evaluation of the singles (10 in the combinations vs. 13 in the singles). The non-significant descriptors for the combinations were 'passion fruit', 'peach', 'apple', 'orange', 'tea', 'honey', 'dusty/mineral', 'cooked veg' and 'tomato leaf'. Descriptors with a significant main or interaction effect were 'guava', 'pineapple', 'banana', 'lemon', 'grapefruit', 'floral', 'orange blossom', 'bergamot/Earl Grey' and 'artificial sweet'.

In the PCA, the high-3MH samples were spread along PC1. Three high-3MH samples (3_1_1, 3_3_1 and 3_2_2) were all associated with 'lemon', 'grapefruit', 'guava' and 'pineapple' (Fig. 4). The two high-3MH samples not in this group also contained high linalool. One of them, 3_3_3, was associated with both 'guava' and 'floral', while the other, 3_1_3, was correlated best with 'floral' (Fig. 4). From the ANOVA of the combinations, and as in the singles, the level of $3 \mathrm{MH}$ had a significant effect on 'guava' and increased at higher $3 \mathrm{MH}$ concentrations, which can be seen in the spider plot for 3_1_1 and 3_3_1 (Fig. 5).

Three-dimensional data representation can be done with the help of surface plots. Instead of individual data points, these plots show the relationship between a dependent variable (in this case, the intensity of an attribute) and two independent variables (the levels of the spiking compounds). Surface plots have been used to illustrate two-way interactions, highlighting the synergistic or masking effect between compounds for a specific attribute (Fig. 6). In the singles, the potential enhancing of 'guava' by ethyl hexanoate or suppressing of 'guava' by linalool was hypothesised. In the combinations, it can be narrowed down to a suppressing effect by linalool (Fig. 6), although the $3 \mathrm{MH}^{*}$ linalool interaction is only significant at $\alpha=0.1$. Benkwitz et al. (2012) also found that, in aroma reconstitution and omission

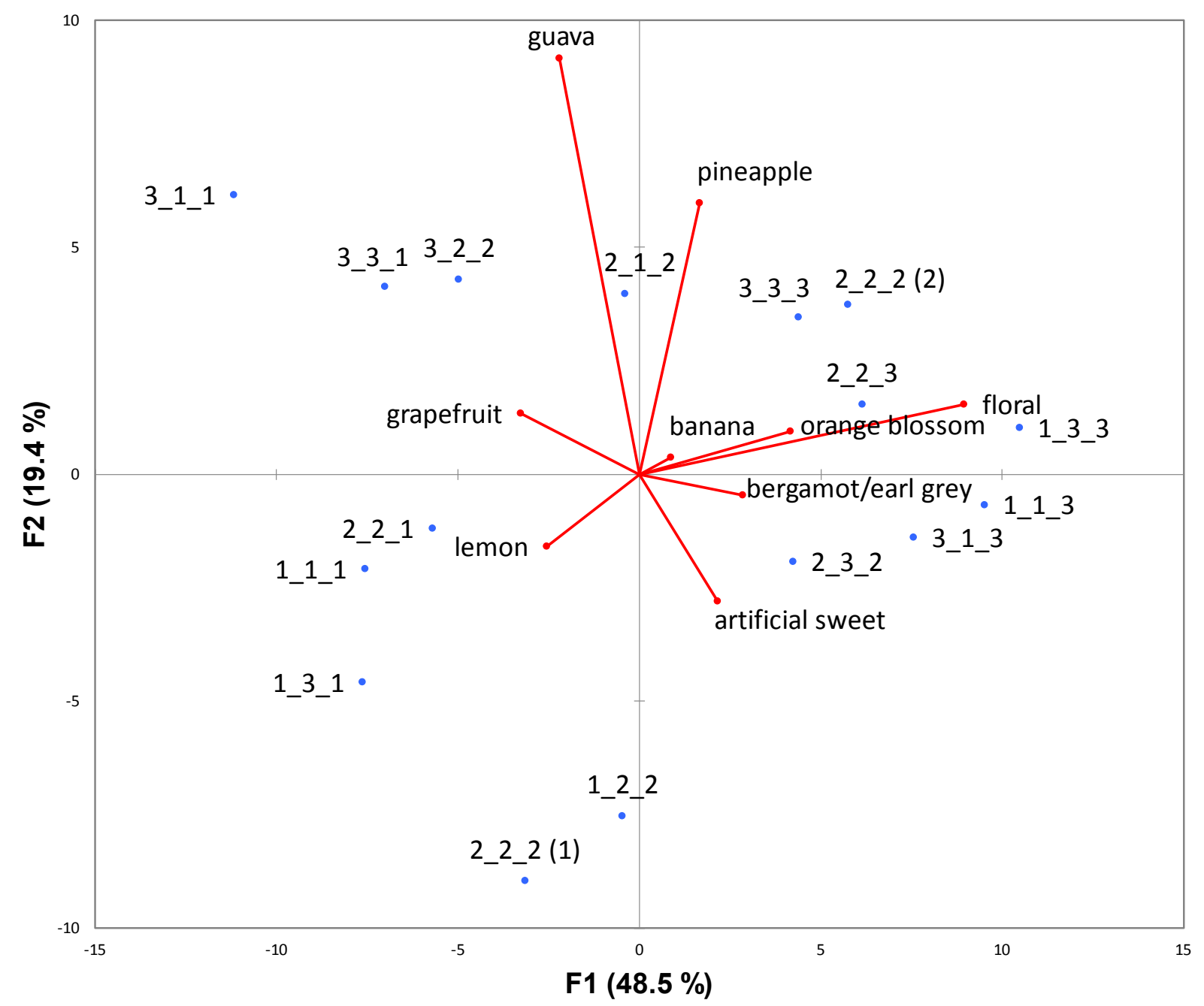

FIGURE 4

PCA of combination data with significant attributes labelled. Sample codes represent the level of 3MH_ethyl hexanoate linalool, where 1 = low, 2 = medium, 3 = high. A full list of sample codes can be found in Table 1 . 


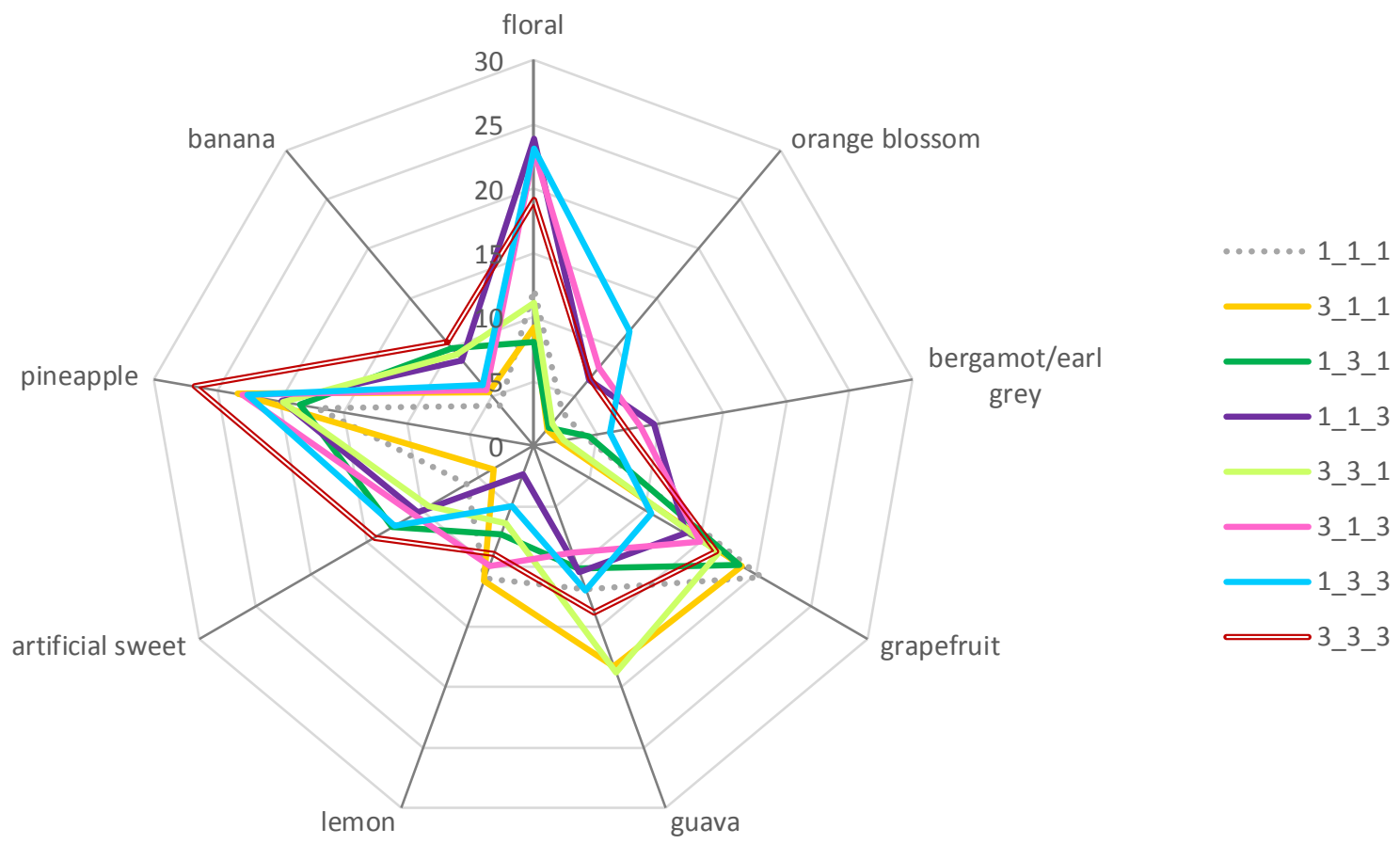

FIGURE 5

Spider web plot showing the aromatic profile of the combinations' DA samples, including only cube (more extreme) samples from the CCD for readability. Includes descriptors with a significant compound main effect or a significant two-compound interaction at $\alpha=0.05$. Sample codes represent the level of 3MH_ethyl hexanoate_linalool, where $1=$ low, $2=$ medium, $3=$ high. A full list of sample codes can be found in Table 1 .

tests of Sauvignon Blanc wines, the omission of linalool led to higher intensities of sweet sweaty passion fruit descriptors, linked to 3MH and 3MHA. In the combinations, 'pineapple' became significant for the $3 \mathrm{MH}$ main effect, while it was not significant in the singles.

'Pineapple' intensity was highest for the sample $3 \_3$ _3 and lowest for 1_1_1 (Fig. 5), so the three compounds seem to have an additive effect for 'pineapple', with $3 \mathrm{MH}$ having the strongest effect. For the descriptor 'tomato leaf', 3 1_ 1 had higher 'tomato leaf' intensity than the other samples (raw data, not shown), which agrees with the significant positive correlation between $3 \mathrm{MH}$ and 'tomato leaf' in the singles. However, there were no significant compound or interaction effects for 'tomato leaf' according to the ANOVA. This indicates that, in the presence of other volatiles, this quality of $3 \mathrm{MH}$ is suppressed. Similarly, the increase in 'cooked veg' due to $3 \mathrm{MH}$ that was observed in the singles was no longer present in the combinations.

'Passion fruit' was not significant in either the singles or combinations at $\alpha=0.05$, but in the combinations there was a trend for the 'passion fruit' intensity to increase at the medium $3 \mathrm{MH}$ concentration $(p=0.054)$. There was also a trend $(p=0.063)$ for linalool and ethyl hexanoate to interact with each other, increasing the 'passion fruit' intensity when both compounds were at high or low concentrations, and decreasing it when both compounds were at medium concentrations (Fig. 6). The last descriptor affected by $3 \mathrm{MH}$ was 'lemon'. In the singles, 'lemon' was suppressed by linalool, but in the combinations there was a significant $3 \mathrm{MH}^{*}$ linalool interaction, with the suppressing effect only exerted when in combination with low $3 \mathrm{MH}$ (Fig. 6). The presence of $3 \mathrm{MH}$ seems to counteract the suppressing effect of linalool on 'lemon'.

All the high-linalool samples were associated with the attributes 'orange blossom', 'floral' and 'bergamot/Earl Grey' attributes in the PCA (Fig. 4). An increase in 'floral' and 'orange blossom' intensities between the low- and high-linalool samples is visible in the spider plot (Fig. 5). From the ANOVA results, 'floral', 'orange blossom' and 'bergamot/Earl Grey' had a significant main effect for linalool, where samples with high linalool concentration were described by these attributes (results not shown). There was a trend for the 'tea' to behave the same way as these descriptors, but it was only significant at $\alpha=0.1$, and not at $\alpha=0.05$. This group of descriptors showed the same behaviour in the singles, showing that these descriptors are a result of linalool, and therefore are not highly enhanced or suppressed by the thiol or the ester. 'Grapefruit' perception decreased significantly at medium and high linalool levels, showing the same suppressing effect that was apparent in the singles (Fig. 7). The suppression of 'cooked veg' by linalool that was observed in the singles is not significant in the combinations at $\alpha=0.05$, but the same behaviour is seen as a trend at $\alpha=0.1$.

The descriptors of which the behaviour is different in the combinations than in the singles in relation to linalool are 'peach', 'lemon' and 'orange'. In the singles, linalool increased 'peach' intensity significantly, but in the combinations there is an interaction that is nearly significant $(p=0.057)$ between ethyl hexanoate and linalool, where 
linalool still increases 'peach' intensity, but only when ethyl hexanoate levels are low. This means that ethyl hexanoate suppresses the 'peach' aroma that is associated with medium and high levels of linalool. 'Lemon' was also affected by an interaction, but between linalool and 3MH (Fig. 6). In the singles, 'lemon' seemed to be a quality of the base wine that was suppressed by linalool. In the combinations, it was suppressed by linalool only when $3 \mathrm{MH}$ levels were low, as high $3 \mathrm{MH}$ levels enhanced 'lemon' intensity. In the combinations, 'orange' intensity was no longer increased by high levels of linalool, just as it was in the singles.

From the PCA of the combinations, it is clear that samples with high ethyl hexanoate were scattered around the PCA, which was expected, considering the subtle effect of the compound seen in the singles (Fig. 3). In the combinations, ethyl hexanoate had a significant effect on 'banana' and 'artificial sweet', which it did not have on the singles. This 'artificial sweet' aroma could be similar to the 'confectionary' aroma given by a combination of esters, including ethyl hexanoate, as described by King et al. (2011). The intensities of both 'artificial sweet' and 'banana' increased significantly with higher levels of ethyl hexanoate (Fig. 7). The fact that 'banana' and 'artificial sweet' were not significant in the singles, coupled with the fact that the intensity of both descriptors was highest for the sample 3_3_3 (Fig. 5), suggests an additive effect similar to that seen with 'pineapple'. These findings are in agreement with other research, in which esters are more likely to support the aromas of other volatiles, rather than contribute as impact compounds on their own (Campo et al., 2005).

\section{CONCLUSIONS}

In the context of the partially dearomatised Chenin Blanc wine matrix, $3 \mathrm{MH}$ was described with attributes previously
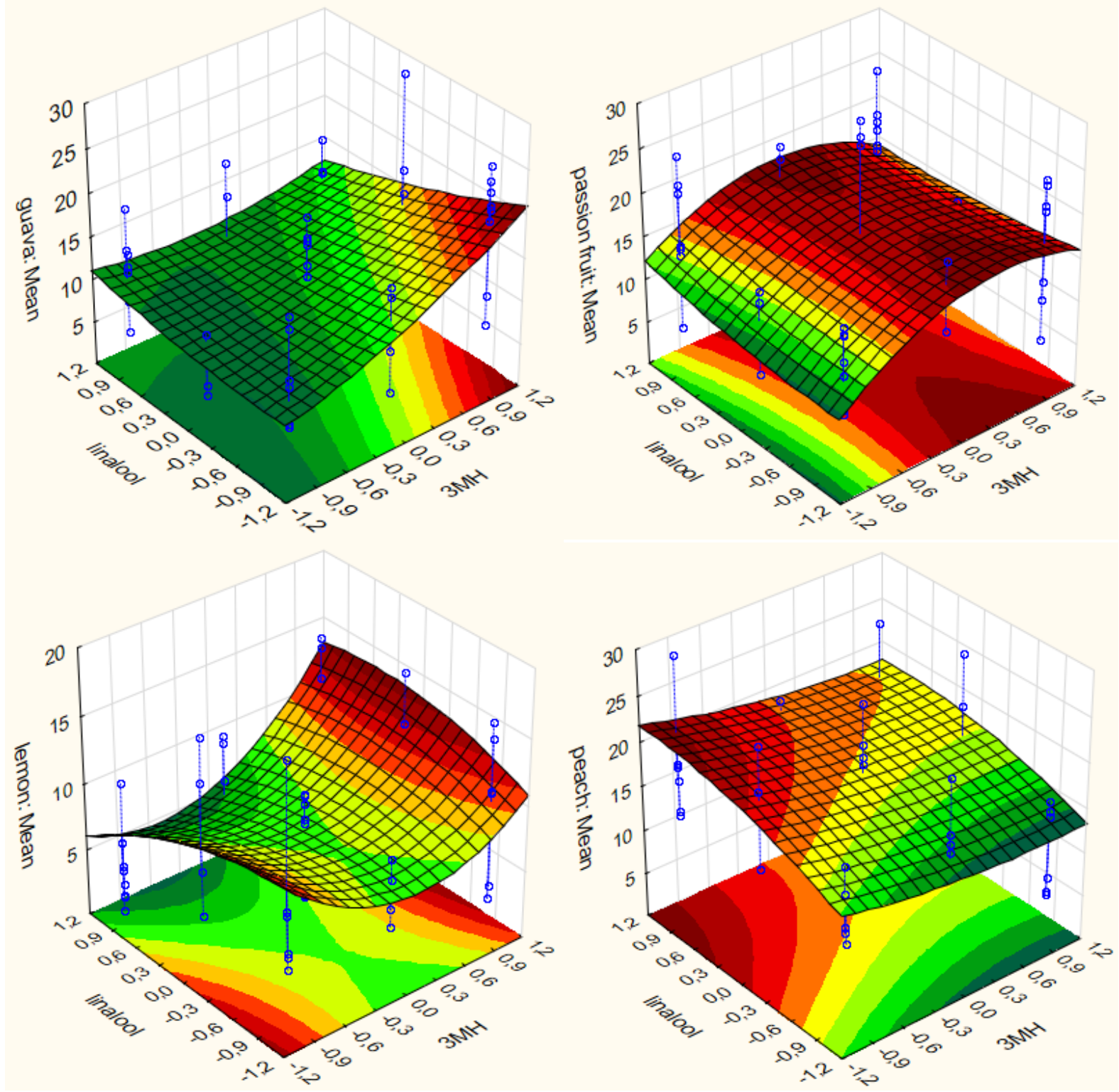

FIGURE 6

Response-surface plot for the intensity of the 'guava', 'passion fruit', 'lemon' and 'peach' attributes due to the interaction of $3 \mathrm{MH}$ and linalool. 
generated for thiols in Sauvignon Blanc wines (Dubourdieu et al., 2006; King et al., 2011; Van Wyngaard et al., 2014; Coetzee et al., 2015). However, unlike the case in Sauvignon Blanc wines, 'passion fruit' and 'grapefruit' intensity did not change significantly at different $3 \mathrm{MH}$ levels. This could either be because the $3 \mathrm{MH}$ range used in this study was narrower than that used in studies on Sauvignon Blanc, or because matrix effects may cause $3 \mathrm{MH}$ to be perceived differently in Chenin Blanc than in Sauvignon Blanc wines.
By following the approach suggested by Van Rooyen et al. (1982), it was found that the most intense aroma of $3 \mathrm{MH}$ was 'guava'. This supports the hypothesis of Du Plessis and Augustyn (1981) that a thiol is responsible for the 'guava' character of Chenin Blanc, although in our case the thiol is $3 \mathrm{MH}$ rather than 4MMP. Research on Sauvignon Blanc has established several ways in which thiols can be manipulated by producers (Coetzee \& Du Toit, 2012), and this information can be used to alter the typical 'guava' and
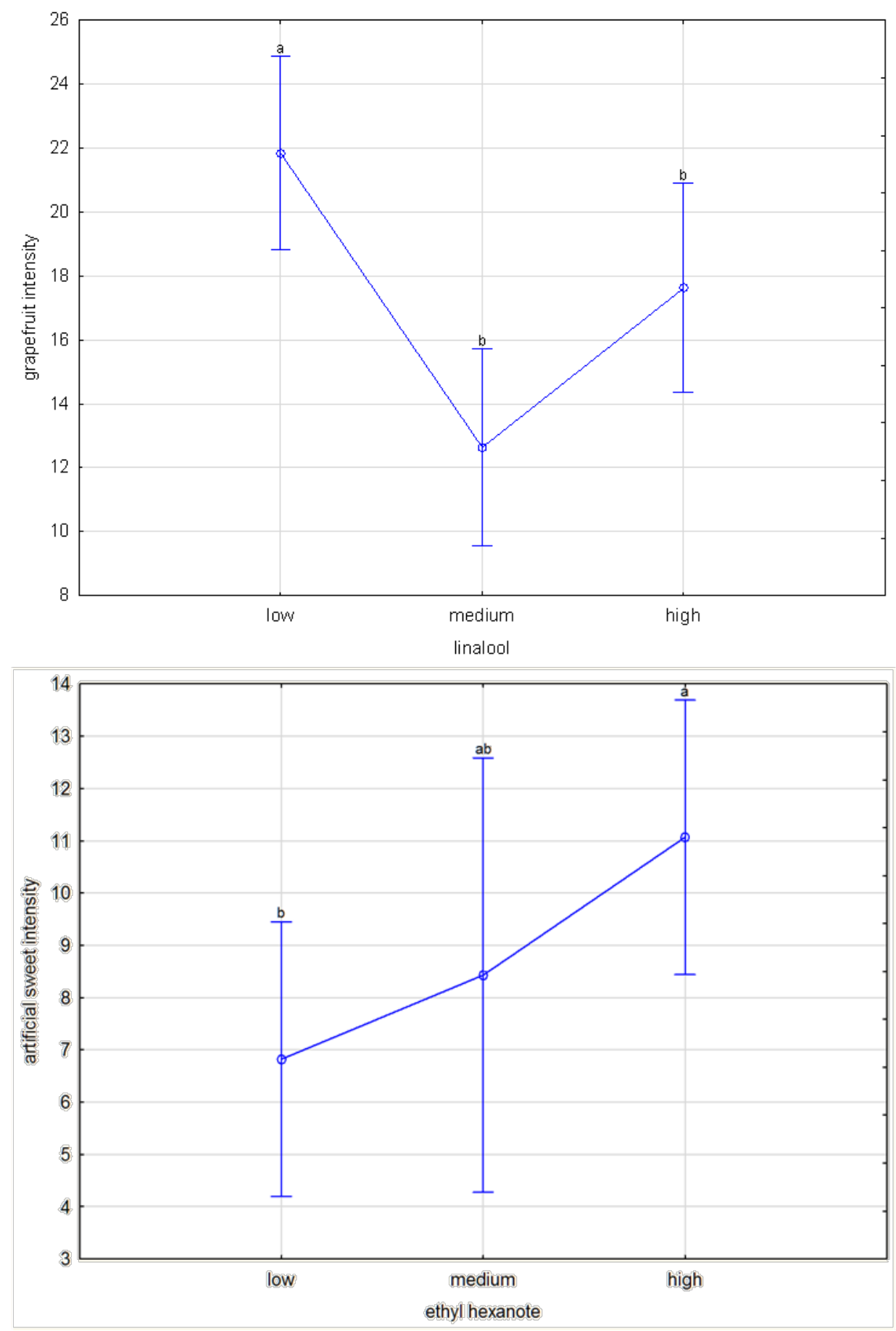

FIGURE 7

LS means plot illustrating the linalool and ethyl hexanoate level effect on 'grapefruit' and 'artificial sweet' aroma intensity for the combinations of compounds with significant letters respectively from LSD post-hoc test. Vertical bars denote $95 \%$ confidence intervals. 
other thiol-derived characters of Chenin Blanc wines.

In the singles, several enhancing and suppressing effects were hypothesised, which were confirmed by the combinations. Most notably, the 'guava' and 'floral' qualities of $3 \mathrm{MH}$ and linalool seem to be antagonistic, which was previously found by Benkwitz et al. (2012). This suggests that, within the sensory characterisation of Chenin Blanc wines, it may be difficult to have a wine that is perceived as both highly 'tropical' and highly 'floral'. This opposition may contribute to the different style categories of South African Chenin Blanc wines.

The strong effects of linalool and $3 \mathrm{MH}$, and the weak effect of ethyl hexanoate, on wine aroma show that the relative sensory contribution of different compounds can not necessarily be predicted by their odour-active values alone. The aromatic properties of linalool were dominant, while the effect of ethyl hexanoate was only apparent when in combination with other compounds. Whether these behaviours are unique to each compound, or whether there are trends within volatile compound classes, warrants further investigation.

The goal of this study was to better understand some of the interactions that occur between volatiles in Chenin Blanc wines. It was shown that the perception of these compounds depends on their concentration and context. The interactions between these compounds are complex, but this type of knowledge can ultimately help researchers better understand the relationship between chemical composition and human sensory perception. Ideally, this study would be expanded to include other volatiles and be replicated in other model solutions to confirm that the sensory responses observed in this study are applicable to other matrices.

\section{LITERATURE CITED}

Benkwitz, F., Nicolau, L., Lund, C., Beresford, M., Wohlers, M. \& Kilmartin, P.A., 2012. Evaluation of key odorants in Sauvignon Blanc wines using three different methodologies. J. Agric. Food Chem. 60, 25, 6293-6302.

Black, C.A., Parker, M., Siebert, T., Capone, D. \& Francis, I. 2015. Terpenoids and their role in wine flavour: Recent advances. Aust. J. Grape Wine Res. 21, 582-600.

Borgognone, M.G., Bussi, J. \& Hough. G., 2001. Principal component analysis in sensory analysis: covariance or correlation matrix? Food Qual. Prefer. 12, 5-7, 323-326.

Campo, E., Ferreira, V., Escudero, A. \& Cacho, J., 2005. Prediction of the wine sensory properties related to grape variety from dynamic-headspace gas chromatography - Olfactometry Data. J. Agric. Food Chem. 53, 56825690 .

Coetzee, C. \& du Toit, W.J., 2012. A comprehensive review on Sauvignon Blanc aroma with a focus on certain positive volatile thiols. Food Res. Int. $45,1,287-298$.

Coetzee, C., Brand, J., Emerton, G, Jacobson, D., Silva Ferreira, A. C. \& du Toit, W. J., 2015. Sensory interaction between 3-mercaptohexan-1-ol, 3-isobutyl-2-methoxypyrazine and oxidation-related compounds. Aust. J. Grape Wine Res. 21, 2, 179-188.

Dubourdieu, D., Tominaga, T., Masneuf, I., Peyrot des Gachons, C. \& Murat, M. L., 2006. The role of yeasts in grape flavor development during fermentation: The example of Sauvignon Blanc. Am. J. Enol. Vitic. 57, 1, $81-88$.
Engel, K.H. \& Tressl, R., 1991. Identification of New Sulfur-Containing Volatiles in Yellow Passion Fruits (Passiflora edulis f. flavicarpa). J. Agric. Food Chem. 39, 12, 2249-2252.

Esbensen, K.H., 2002. Multivariate Data Analysis - in practice. (5th ed.). CAMO Process AS, Oslo, Norway.

Ferreira, V., Lopez, R. \& Cacho, J.F., 2000. Quantitative determination of the odorants of young red wines from different grape varieties. J. Sci. Food Agric. 80, 11, 1659-1667.

Francis, I.L. \& Newton, J.L., 2005. Determining wine aroma from compositional data. Aust. J. Grape Wine Res. 11, 2, 114-126.

Fretz, C.B., Luisier, J.L., Tominaga, T. \& Amadò, R., 2005. 3-Mercaptohexanol: An aroma impact compound of Petite Arvine wine. Am. J. Enol. Vitic. 56, 4, 407-410.

ISO NORM, n.d. 3591:1977: Sensory analysis: Apparatus wine tasting glass. International Organization for Standardization Geneva, Switzerland.

ISO NORM, n.d. 8589:2007: Sensory analysis: General guidance for the design of test rooms. Geneva, Switzerland.

King, E.S., Osidacz, P., Curtin, C, Bastian, S.E.P. \& Francis, I.L., 2011. Assessing desirable levels of sensory properties in Sauvignon Blanc wines - consumer preferences and contribution of key aroma compounds. Aust. J. Grape Wine Res. 17, 2, 169-180.

Lawless, H.T. \& Heymann, H., 2010. Sensory Evaluation of Food Principles and Practices. (2nd ed.). Springer, New York.

Lawrence, N., 2012. Volatile metabolic profiling of SA Chenin Blanc fresh and fruity and rich and ripe wine styles : Development of analytical methods for flavour compounds ( aroma and flavour) and application of chemometrics for resolution of complex analytical measurement.

López, R., Ortín, N., Perez-Trujillo, J.P., Cacho, J. \& Ferreira, V., 2003. Impact odorants of different young white wines from the Canary Islands. J. Agric. Food Chem. 51, 11, 3419-3425.

MacFie, H., Bratchell, N., Greenhoff, K. \& Vallis, L.V., 1989. Designs to balance the effect of order of presentation and first-order carry-over effects in hall tests. J. Sens. Stud. 4, 2, 129-148.

Marais, J., 1983. Terpenes in the aroma of grapes and wines. S. Afr. J. Enol. Vitic. 4, 2, 49-58.

Mateo-Vivaracho, L., Zapata, J., Cacho, J. \& Ferreira, V., 2010. Analysis, occurrence, and potential sensory significance of five polyfunctional mercaptans in white wines. J. Agric. Food Chem. 58, 18, 10184-10194.

Mateo, J.J. \& Jiménez, M., 2000. Monoterpenes in grape juice and wines. J. Chromatogr. A 881, 1-2, 557-567.

Padrayauttawat, A., Yoshizawa, T., Tamura, H. \& Tokunaga, T., 1997. Optical Isomers and Odor Thresholds of Volatile Constituents in Citrus sudachi. Food Sci. Technol. Int. 3, 4, 402-408.

Piano, F., Fracassetti, D., Buica, A., Stander, M., du Toit, W.J., Borsa, D. \& Tirelli, A., 2015. Development of a novel liquid/liquid extraction and ultraperformance liquid chromatography tandem mass spectrometry method for the assessment of thiols in South African Sauvignon Blanc wines. Aust. J. Grape Wine Res. 21, 1, 40-48.

Pino, J.A. \& Bent, L., 2013. Odour-active compounds in guava (Psidium guajava L. cv. Red Suprema). J. Sci. Food Agric. 93, 12, 3114-3120.

Du Plessis, C.S. \& Augustyn, O.P.H., 1981. Initial study on the guava aroma of Chenin Blanc and Colombar wines. S. Afr. J. Enol. Vitic 2, 2, 101.

Polášková, P., Herszage, J.\& Ebeler, S.E., 2008. Wine flavor: chemistry in a glass. Chem. Soc. Rev. 37, 11, 2478-2489. 
Steinhaus, M., Sinuco, D., Polster, J., Osorio, C. \& Schieberle, P., 2009. Characterization of the key aroma compounds in pink guava (Psidium guajava L.) by means of aroma re-engineering experiments and omission tests. J. Agric. Food Chem. 57, 7, 2882-2888.

Swiegers, J.H. \& Pretorius, I.S., 2007. Modulation of volatile sulfur compounds by wine yeast. Appl. Microbiol. Biotechnol. 74, 5, 954-960.

Tomic, O., Luciano, G., Nilsen, A., Hyldig, G, Lorensen, K. \& Næs, T., 2009. Analysing sensory panel performance in a proficiency test using the PanelCheck software. Eur. Food Res. Technol. 230, 3, 497-511.

Tominaga, T., Furrer, A., Henry, R. \& Dubourdieu, D., 1998. Identification of new volatile thiols in the aroma of Vitis vinifera L. var. Sauvignon Blanc wines. Flavour Fragr. J. 13, 3, 159-162. van Rooyen, P.C., de Wet, P., et al., 1982. Chenin Blanc wine volatiles and the intensity of a guava-like flavour. S. Afr. J. Enol.Vitic. 3, 1, 1-7.

van Wyngaard, E., Brand, J., Jacobson, D. \& du Toit, W.J., 2014. Sensory interaction between 3-mercaptohexan-1-ol and 2-isobutyl-3methoxypyrazine in dearomatised Sauvignon Blanc wine. Aust. J. Grape Wine Res. 20, 2, 178-185.

Wilson, C.L., 2016. Chemical evaluation and sensory relevence of thiols in South African Chenin Blanc wines. MSc Thesis. Stellenbosch University. 